\title{
PCA BAND SELECTION METHOD FOR A HYPERSPECTRAL SENSORS ONBOARD AN UAV
}

\author{
J. A. S. Centeno ${ }^{1}$, J. Kern ${ }^{2}$, E.A. Mitishita ${ }^{1}$, M.E.J. Palma ${ }^{1}$ \\ ${ }^{1}$ UFPR Geomatics Department, UFPR, Curitiba, Brazil - (centeno, mitishita)@ufpr.br, majijpa@ hotmail.com \\ ${ }^{2}$ Institut for Photogrammetry and Remote Sensing - KIT, Karlsruhe, Germany - jens.kern@kit.edu
}

KEY WORDS: Hyperspectral remote sensing, feature selection, UAV

\begin{abstract}
:
The development of light and small sensors, like Lidar and hyperspectral sensors, has gained popularity over the last few years. In this paper we present the experience of UFPR (Brazil), in collaboration with KIT (Germany), on the use of a UAV system carrying a hyperspectral sensor for land cover studies. The sensors were integrated with the traditional IMU-GNSS systems to record data from a quadricopter. The study focuses on band selection, aiming at reducing computational effort and statistical limitations. For this purpose, the principal components of the multispectral image are computed. The best principal components are then selected according to the explained original variance, as described by the relative size of the eigenvalues. Then, each principal component is analyzed searching for contrasting spectral regions, described by consecutive positive and negative coefficients. The most representative band of each spectral region is the selected according to its information contents and contribution to the computation of the respective eigenvectors. The method is tested using images collected with the FireflEYE 185 Cubert camera with 125 channels in the wavelength between $450 \mathrm{~nm}$ and $950 \mathrm{~nm}$, flying over the experimental Canguiri farm in Curitiba, Brazil. Finally, we discuss the advantages of the method and its limitations.
\end{abstract}

\section{INTRODUCTION}

The use of UAV (unmanned aerial vehicles), supported by advances in image processing and photogrammetry, is well established in Cartography. This practice is only possible because of the reduction of imaging devices size, weight and energy consumption, as well as advances in battery size and power(Colomina and Molina, 2014). As Kelcey and Lucieer (2012) point out, UAV technology can be not as a concurrent for remote sensing, but more as a complementary tool, because it provides a detailed view of the study area with high spatial resolution. The cartographic role of UAV has increased in the last decade. In 2017, the ISPRS organized the International Conference on Unmanned Aerial Vehicles in Geomatics in Bonn, showing that this practice gained relevance within the research fields of photogrammetry and remote sensing.

As new sensors are developed and improved, the possibilities for UAVs increase. The common practice of carrying cameras is no more restricted to the visible spectrum, but even hyperspectral sensors are currently available. hyperspectral remote sensing offers the availability of more than a hundred of narrow spectral bands. Hyperspectral remote sensing is based on the measurement of electromagnetic radiation in a high number of narrow, contiguous spectral bands. In this context, the narrowness and contiguous nature of the measurements is more relevant than the number of bands.

The main advantages provided by hyperspectral sensors are related to identifying materials according to a detailed spectral description of the surface (surface material identification) and to distinguishing between spectrally similar surfaces (Material differentiation). Both practices are part of general mapping and monitoring activities. For example, concerning vegetation studies, it becomes possible to map species ( $\mathrm{Li}$ et al, 2017) and monitoring plant health (e.g., Gerhards et al.,2019).

Hyperspectral image interpreting requires the understanding of the detailed spectral properties of the materials and can be strongly affected by atmospheric disturbances, illumination changes and spectral mixing. The high number of spectral bands increases detail and information (Benediktsson and Ghamisi 2015), but it also may reduce the classification accuracy as the number of bands increases too much. This problem is known as the Hughes' phenomenon (Hughes 1968), and is caused by the limited number of samples versus the number of available bands.

There are two approaches for dimensionality reduction (Serpico et al. 2003): selection of the most relevant subset of spectral bands (feature selection) and computation of a reduced set of new significant variables, combination of the original ones, (feature extraction). Examples of the first approach are described in Su et al. (2016) or Fauvel et al. (2015). To the second group belongs the work of Ren et al. (2014) or Zhong et al. (2015). Ren et al. (2014) proposed the use of the Modified Principal Components method to reduce the dimensionality. This method consists of maintaining locally structured elements which only appear in a small number of bands to improve the discrimination when feature bands are extracted as principal components.

In this paper it is introduced a feature extraction approach to reduce the dimensionality of hyperspectral data by analyzing the coefficients of the eigenvectors computed from the variancecovariance matrix of the hyperspectral image. Although this method is based in the principal components feature extraction method, it introduces a novel band selection approach that is based on the analysis of the series of coefficients and the identification of the most relevant bands considering the contribution of each band to the principal components set and the variance explained by the principal components.

Principal Components Transform (PC) is widely used to analyse hyperspectral images, because this technique allows computing a reduced uncorrelated set of variables that are linear combination of the original ones. The special property of the Principal Component Transform is the fact that the small set of computed variables stores almost all the explainable variance of the original set. In this sense, a small set of variables can be 
used to represent the original data with a minimal loss of information.

Although the Principal Component Transform provides a set of uncorrelated variables, their computation requires the use of all available bands, including spectral neighbouring bands that are highly correlated. This article discusses a variation of the use of the Principal Components Transform for feature selection when dealing with a very high number of contiguous spectral bands collected using a hyperspectral sensor on an UAV (Unmanned Aerial Vehicle). The difference between using hyperspectral data collected from a satellite and using an UAV is mainly the reduction of the atmospheric effects because the UAV is very close to the surface. Therefore, absorption bands like those caused by water or ozone are not present in the UAV data and the spectral series is more continuous.

\section{METHOD}

To compute the coefficients of the Principal Components Transform it is necessary to compute the variance-covariance matrix of the hyperspectral image. For example, using an image with $n b$ bands require the computation of a $n b \times n b$ covariance matrix $\operatorname{VC}(n b \times n b)$.

The covariance matrix stores information about the interdependency between the spectral bands that can be estimated by the correlation. The correlation matrix can be computed as:

$$
M C(i, j)=\frac{V C(i, j)}{\operatorname{std}(i) * \operatorname{std}(j)}
$$

With $i$ and $j=1 \ldots n$, where $n$ is the number of spectral bands.

As the variance-covariance matrix is a real, positive, semidefinite matrix it is possible to compute its eigenvalues $(\lambda)$ that are always greater than or equal to zero, according to equation 2.

$$
\operatorname{det}(V C-\lambda I)=0
$$

Finally, the eigenvectors associated to each computed eigenvalue $\lambda$ are the vectors $v$ that satisfy:

$$
V C \cdot v=\lambda \cdot v
$$

The eigenvalues and eigenvetors can be used to compute the principal components, according to Equation 4.

$$
\mathrm{PC}=\mathrm{X} * \mathrm{E}
$$

Where $\quad \mathrm{X}:\left(\begin{array}{llll}\mathrm{x}_{1} & \mathrm{x}_{2} & \mathrm{x}_{3} \ldots & \mathrm{x}_{\mathrm{nb}}\end{array}\right)=$ original variables $($ spectral bands).

$\mathrm{CP}:\left(\mathrm{CP}_{1} \mathrm{CP}_{2} \mathrm{CP}_{3} \ldots \mathrm{CP}_{\mathrm{nb}}\right)=$ transformed features

$E=n b \times n b$ matrix where each column stores an eigenvector.

The sum of the eigenvalues is equal to the total variance of the original set. The variance explained by each principal component is given by the ratio between the size of the associated eigenvalue and the total variance, as shown in Equation 5.

$$
a_{i}=\frac{\lambda_{i}}{\sum_{j=1}^{n} \lambda_{j}}
$$

$$
\text { with } \quad i=1,2,3 \ldots n b
$$

The component related to the highest $a_{i}$ (equation 4), is called the first principal component. Sorting the components according to the explained variance $\left(a_{i}\right)$ allows identifying the main (principal) components and discarding the less important.

The ith-component can be computed as:

$$
C P_{i}=\sum_{j=1}^{n} v_{i j} \cdot x_{j}
$$

Where $\mathrm{v}_{\mathrm{ij}}$ is the $j$ th-coefficient of the ith-eigenvector and $\mathrm{x}_{\mathrm{j}}$ is the digital value in the $j t h$-spectral band.

The relative sizes of the coefficients provide information about the relative importance of the associated variable. Figure 1 displays an example of the coefficients of the first principal component of a real image. There are positive coefficients for lower bands and negative coefficients higher bands. So, it can be deduced that the component reflects the contrast between two spectral regions. Nevertheless, the use of a weighted sum of all bands in the first spectral region compared to the weighted sum of the bands in the second region includes redundant information, as spectrally close bands are highly correlated. Therefore, instead of using the weighted sum, we propose the use of a representative band, the one associated to the maximum of each region, one for the visible and one for the near infrared, according to the computed weight.

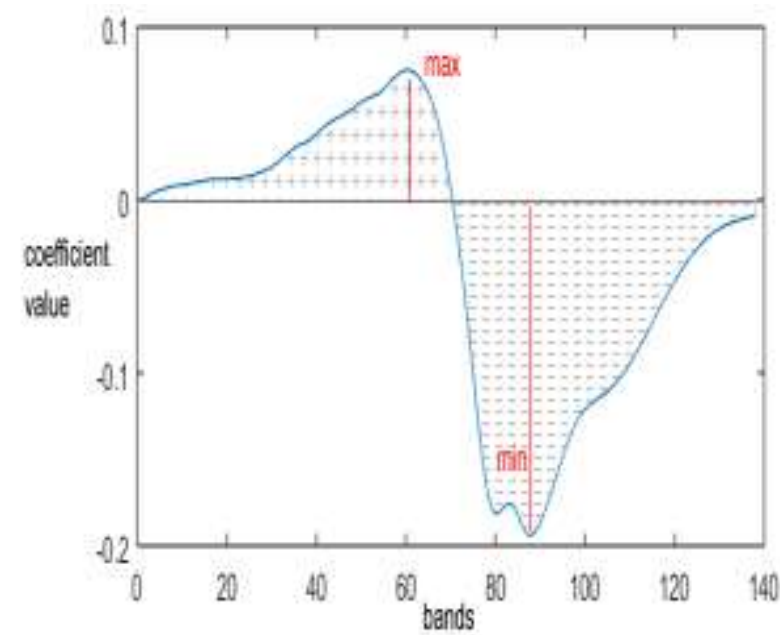

Figure 1. Example of a series of coefficients used to compute a principal component (eigenvalue coefficients).

A function is used to estimate the principal component weights variations along the spectrum $F(X)$ and its local minima and maxima are computed. These points are located at $x$ values that have zero slope (equation 7)

$$
F^{\prime}=\frac{d(F)}{d(x)}=0
$$

The value of the second derivate gives information about the type of extreme point, maximum or minimum.

$F^{\prime \prime}=\frac{d\left(F^{\prime}\right)}{d(x)}=\left\{\begin{array}{l}>0 \text { minimum } \\ <0 \text { maximum }\end{array}\right.$ 
So, selecting the local minima and maxima it is possible to detect the most significant spectral bands used in each component.

The relative contribution of the selected variable to the computation of the principal component is given by its size compared to the sum of the absolute value of all coefficients, as shown in equation 9.

$$
b_{i}=\frac{\left|v_{i}\right|}{\sum_{j=1}^{n}\left|v_{j}\right|}
$$

Considering Figure 2, where the absolute value of the coefficients is represented, the sum of all coefficients is equal to the area between the blue curve and the $\mathrm{x}$-avis. Now, if two coefficients are selected to represent this component, it would be reasonable to estimate their contribution as the sum of all neighbouring coefficients that each one represents. For example, in Figure 2, the relative weight of $V_{i}$ would be equal to the gray area divided by the total sum of the coefficients (all areas).

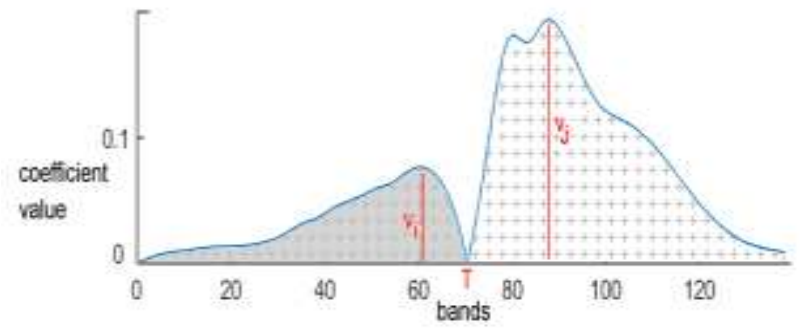

Figure 2. Example of the absolute value of a series of coefficients.

$$
w_{i}=\frac{\sum_{j=1}^{T} v_{j}}{\sum_{j=1}^{n} v_{j}}
$$

Finally, the total contribution ( $c o$ ) of a selected band is given by its contribution to the computation of the component $(w)$ weighted by the contribution of the component $(a)$

$$
c o_{i j}=a_{i} \cdot w_{i j}
$$

This value can be used to select the most relevant bands within a given set of hyperspectral bands.

\section{MATERIAL AND STUDY AREA}

To evaluate the proposed method hyperspectral data were obtained using a FireflEYE 185 Cubert GmbH camera with 125 channels in the wavelength range from $450 \mathrm{~nm}$ to $950 \mathrm{~nm}$. The camera takes hyperspectral images in a 50x50 frame and also a $100 \times 100$ panchromatic image. The camera was installed on a UAV (coaxial octocopter) that flew over the experimental Canguiri farm of the Federal University of Paraná. The sensor was mounted on a two axis gimbal. Ancillary devices were used to support the navigation, such as a RTK GNSS system. The FireflEYE 185 collects images in the wavelength range between 450-950nm using 125 bands.

\section{RESULTS}

To illustrate the process and the results, we use a small 50x50 image with 125 bands, as displayed in Figure 3. Figure 3a displays a RGB combination obtained over a crop field. Part of a road (right) and a crop field (left) are visible in the image. Figure $3 b$ displays the correlation matrix of the image, transformed into a 3D grid. It is interesting to note that there are some regions of low covariance values around the 70 th $-80^{\text {th }}$ spectral band. Low correlation values are found at the extremes of the matrix, where the bands are spectrally far.

Figure $3 \mathrm{a}$ also displays the location of two soil pixels and three vegetation pixels over a false-colour composite. The digital values of these pixels were read and are displayed in figure 4 . It is visible that the digital values of vegetation are lower than those of soil in the first spectral bands (visible) but are higher in the last ones (near infrared). Therefore, there is an inversion around $735 \mathrm{~nm}$ that is also visible in the correlation matrix.

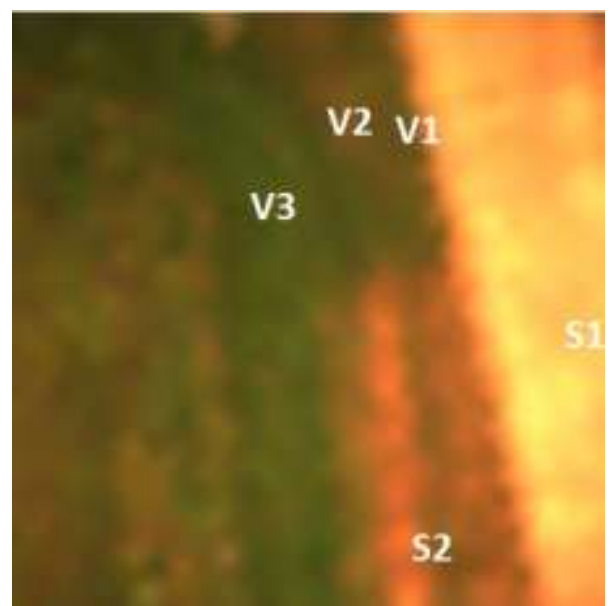

(a)

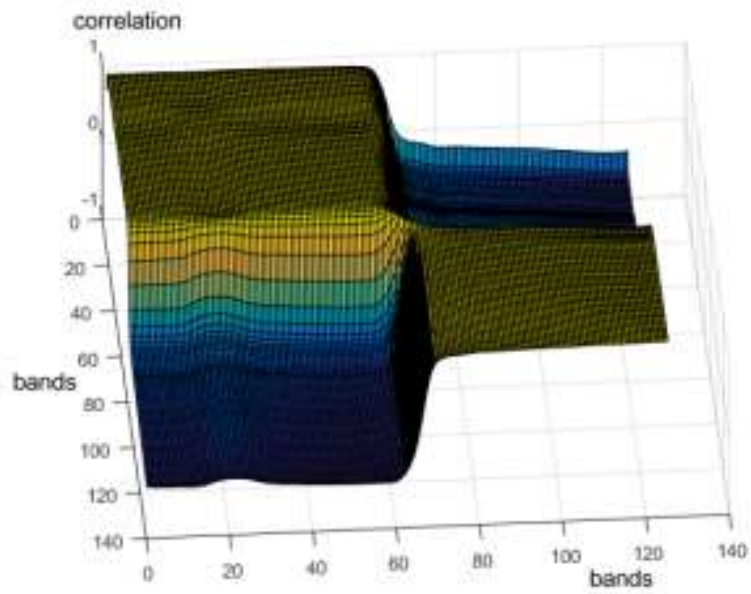

(b)

Figure 3. Example of a hyperspectral image (RGB composition) and its correlation matrix. 


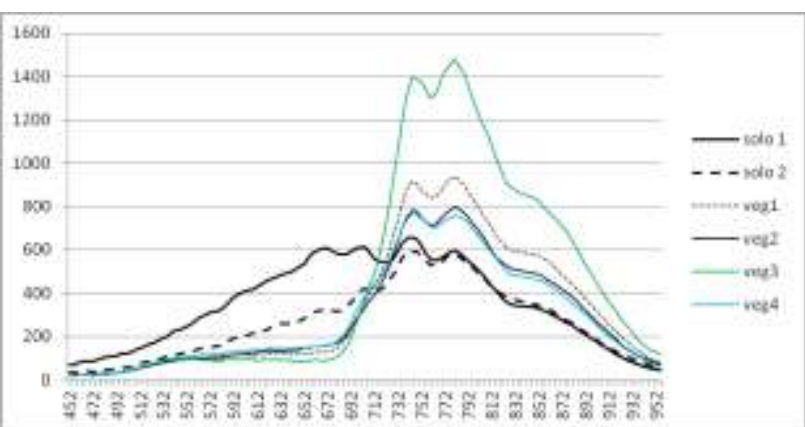

Figure 4. Digital values of selected pixels

Now, after computing the eigenvalues and eigenvectors, it is possible to sort the principal components according to the explained variance. In the example, six components are enough to explain $99,9 \%$ of the original variance, as shown in Table 1 .

\begin{tabular}{|c|c|c|c|}
\hline Component & Eigenvalue & Percent & Cum. Percent \\
\hline 1 & 1790820,846 & 81,96 & 81,96 \\
2 & 378840,9971 & 17,34 & 99,30 \\
3 & 7668,3801 & 0,35 & 99,65 \\
4 & 4344,3382 & 0,20 & 99,85 \\
5 & 889,3248 & 0,04 & 99,89 \\
6 & 373,1741 & 0,02 & 99,91 \\
\hline
\end{tabular}

Table 1. Variance explained by the first components of a hyperspectral UAV image.

To illustrate the method, only the first four components will be analyzed. This will avoid the pollution of the graphs. Figure 5 displays the coefficients of the first four principal components. The first component displays the contrast between the visible and infrared. The second one is more the sum of all bands, giving emphasising the red/red-edge region. The third component shows the contrast between the red-edge and the other bands and the fourth one involves four spectral regions.

The red dots in figure 5 show the local minima and maxima computed using the proposed approach. The selected bands are located at: $\left[\begin{array}{llllll}560 & 688 & 740 & 772 & 800 & 916\end{array}\right] \mathrm{nm}$.

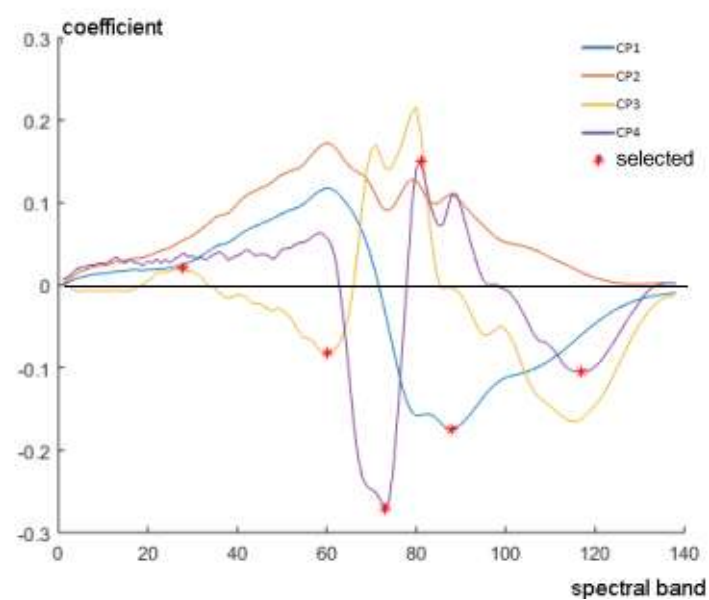

Figure 4. Selected spectral bands overlaid on the coefficient series.

\subsection{Verification}

It is expected that the set of selected bands has lower correlation, since it was derived from the Principal Components. Therefore, the correlation values are displayed in table 2 . The correlation values are mostly low, but some higher correlations are present and highlighted in bold. Traditionally, spectral close bands are correlated and this not a surprise. The positive side is that the selected set includes uncorrelated bands, with very low correlation, almost null in some cases.

\begin{tabular}{|c|c|c|c|c|c|c|}
\hline Bands & 28 & 60 & 73 & 81 & 88 & 117 \\
\hline 28 & 1 & & & & & \\
60 & $\mathbf{0 , 9 7}$ & 1 & & & & \\
73 & 0,13 & $-0,05$ & 1 & & & \\
81 & $-0,43$ & $-0,6$ & 0,78 & 1 & & \\
88 & $-0,48$ & $-0,64$ & 0,75 & $\mathbf{0 , 9 9}$ & 1 & \\
117 & $-0,62$ & $-0,74$ & 0,63 & 0,94 & $\mathbf{0 , 9 6}$ & 1 \\
\hline
\end{tabular}

Table 2. Corrleation between the selected spectral bands.

To assess how the overall correlation was reduced, the mean correlation was computed for the original and for the selected set of bands.

$$
\text { meanC }=\frac{\sum_{i=1}^{n b} \sum_{j=1}^{n b} \operatorname{acorr}(i, j)}{N V}, \text { with } i \neq j
$$

Where

$n b=$ number of spectral bands;

$\operatorname{acorr}(i, j)=$ absolute value of the correlation between band $i$ and band $j$;

$N V=$ number of non zero correlation coefficients.

In the original set there were 125 spectral bands and the mean correlation was 0.778 . When only the selected bands are considered ( 6 bands), the mean correlation is lower, 0.648 . This is a reduction of $16,76 \%$.

\section{CONCLUSION}

The use of hyperspectral images obtained from UAVs faces the challenge of dealing with a great amount of data. In this paper it was introduced a feature extraction approach based on the Principal Component Transform and the analysis of the eigenvectors coefficients. Although the Principal Component Transform provides a set of uncorrelated variables, their computation requires the use of all available bands, including spectral neighbouring bands that are highly correlated. The proposed method is a variation of the use of the Principal Components Transform for feature selection when dealing with a very high number of contiguous spectral bands.

The identification of selected bands according to the variation of the coefficients of the eigenvectors summarizes the main trend that the principal component traduces, but with very few spectral bands.

The use of a weight that is derived from the relative size of the coefficient within the principal component formula and the relative size of the eigenvalue is also a valid option that guides the selection.

As this is the first experiment with the proposed method, more experiments are necessary, including other hyperspectral images. 


\section{REFERENCES}

Kelcey, J., Lucieer, A., 2012. Sensor correction and radiometric calibration of a 6-band multispectral imaging sensor for UAV remote sensing. ISPRS - Int. Arch.Photogramm. Remote Sens. Spatial Inform. Sci. XXXIX-B1, 393-398.

Benediktsson, J. A., Ghamisi P., 2015. Spectral-Spatial Classification of Hyperspectral Remote Sensing Images. Artech House Publishers, INC, Boston, USA.

Colomina, I., Molina, P., 2014. Unmanned Aerial Systems for Photogrammetry and Remote Sensing: A Review. ISPRS Journal of Photogrammetry and Remote Sensing, 92, 79-97.

Serpico, S. B., D’Inca, M., Melgani, F., Moser, G., 2003 Comparison of feature reduction techniques for classification of hyperspectral remote sensing data, International Symposium on Remote Sensing, 4885, pp. 347-35.

Su, H., Yong, B., Du, Q. , 2016. Hyperspectral Band Selection Using Improved Firefly Algorithm. IEEE Geoscience and Remote Sensing Letters, 13(1), 68-72.

Fauvel, M., Dechesne, C., Zullo, A.; Ferraty, F., 2015. Fast Forward Feature Selection ofHyperspectral Images for Classification With Gaussian Mixture Models. IEEE Journal of Selected Topics in Applied Earth Observations and Remote Sensing, 8(6), 2824-2831.

Ren, J.; Zabalza, J.; Marshall, S.; Zheng, J. , 2014. Effective feature extraction and data reduction in remote sensing using hyperspectral imaging. IEEE Signal Processing Magazine, 31(4), 149-154.

Zhong, Z.; Fan, B.; Duan, J.; Wang, L.; Ding, K.; Xiang, S.; Pan, C. , 2015. Discriminant tensor spectral-spatial feature extraction for hyperspectral image classification. IEEE Geoscience and Remote Sensing Letters, 12(5), 1028-1032.

Gerhards, M., Schlerf, M., Mallick, K., Udelhoven, T., (2019). Challenges and Future Perspectives of Multi-/Hyperspectral Thermal Infrared Remote Sensing for Crop Water-Stress Detection: A Review. Remote Sensing 11 (10), 1240.

Yang, C., Everitt, J.H., Bradford, J.M., Murden, D., 2004. Airborne hyperspectral imagery and yield monitor data for mapping cotton yield variability. Prec. Agric. 5 (5), 445-461.

Li, Q. S., Wong, F. K. K., Fung, T., 2017. Assessing the Utility of Uav-Borne Hyperspectral Image and Photogrammetry Derived 3d Data for Wetland Species Distribution Quick Mapping. International Archives of the Photogrammetry, Remote Sensing and Spatial Information Sciences, Volume XLII-2/W6, 2017, pp.209-215.

\section{ACKNOWLEDGEMENTS (OPTIONAL)}

The authors would like to thank CNPq CNPq (Process: 303279/2018-4), DAAD and CAPES for the financial support and the MUDAK (BR/GER) project team for the help. 